\title{
Improved clinical outcomes and physical activity in patients with rheumatoid arthritis treated with originator adalimumab in routine clinical practice
}

Background/Objective: We aimed at evaluating changes in habitual physical activity, disease activity and physical function following treatment with originator adalimumab ( ADA $_{\mathrm{ORIG}}$ ) in patients with rheumatoid arthritis (RA) in routine clinical practice.

Methods: This observational study followed RA patients for 12 months, using the DAS28, HAQ-DI and Short QUestionnaire to ASsess Health-enhancing physical activity (SQUASH) instruments to measure disease activity, physical function and habitual physical activity, respectively. Associations of SQUASH scores with DAS28, HAQ-DI and socio-demographic factors were evaluated by correlation coefficients and multivariable regression analyses.

Results: From baseline to final visit, the mean SQUASH total score increased from $4772 \pm 4132$ to $6104 \pm$ 4921 ( $n=460)$, representing a $28 \%$ improvement in overall physical activity. Largest improvements were observed in the domains of leisure time and sport (42\%), and activities at work and school (35\%). Mean DAS28 and HAQ-DI scores decreased by 2.8 and 0.7 points, respectively, but a sizeable negative correlation was found only between baseline SQUASH total score and HAQ-DI score $(-0.30 ; p<0.0001)$. Advancing age was negatively associated with the final SQUASH total score (effect estimate of $-46.2, p<0.05$ ).

Conclusion: Improvements in habitual physical activity, disease activity, and physical function were observed in RA patients after 12 months of ADA $_{\text {ORIG }}$ therapy.

Keywords: originator adalimumab • rheumatoid arthritis • physical activity • SQUASH scores

\section{Introduction}

Rheumatoid arthritis (RA) is a chronic inflammatory immune-mediated disease characterized by progressive symmetric polyarthritis with subsequent cartilage and bone destruction [1]. Joint pain, stiffness and articular damage impair patients' daily physical functioning [2], which impacts negatively on their autonomy and work ability [3]. Despite exercise being considered beneficial and safe for RA patients, this group is less physically active than the general population [4-6]. Moreover, compared with the general population, RA patients also have a reduced health-related quality of life (HRQoL) [7], and are at greater risk of cardiovascular morbidity and mortality due to cumulative inflammatory burden and multiple other risk factors [8-10].

The benefits of exercise in RA patients have been well documented. Exercise has been shown to have positive effects on aerobic capacity and muscle strength [11], and to improve physical function [12] and HRQoL [13,14] without exacerbating disease activity [12]. A long-term exercise program in RA patients lowered C-reactive protein levels compared with controls [15], with significant improvements apparent in microvascular and macrovascular function as early as 3 months into the program [16].

Together with optimal control of disease activity, the European League Against Rheumatism (EULAR) guidelines recommend regular Physical Activity (PA), a healthy diet and smoking cessation in RA patients to reduce cardiovascular risk [17]. In 2018, EULAR formally advocated PA as an integral part of standard care for persons with inflammatory arthritis (RA and spondyloarthritis) and osteoarthritis and issued specific recommendations about the delivery and evaluation of PA interventions [18].

\section{Dmitry Karateev', Bernadette Rojkovich², Maja Hojnik ${ }^{3}$, Orsolya Nagy ${ }^{* 4}$ \& Cătălin Codreanu ${ }^{5}$}

'Moscow Regional Research and Clinical Institute, Moscow, Russian Federation

2Polyclinic of the Hospitaller Brothers of St. John of God, Budapest, Hungary

${ }^{3}$ Global Medical Affairs, AbbVie, Ljubljana, Slovenia

${ }^{4}$ Global Medical Affairs, AbbVie, Budapest, Hungary

${ }^{5}$ "Dr. Stoia" Center for Rheumatic Diseases, University of Medicine and Pharmacy "Carol Davila", Bucharest, Romania

*Author for correspondence: orsolya.nagy@abbvie.com 
Biologic disease-modifying antirheumatic drugs have significantly improved the pharmacological treatment of RA [19-21]. Originator adalimum$\mathrm{ab}\left(\mathrm{ADA}_{\mathrm{ORIG}}\right)$ is a fully human Tumor Necrosis Factor (TNF) inhibitor monoclonal antibody which, in combination with methotrexate, has been shown in randomized controlled trials (RCTs) to improve outcomes in patients with RA as measured by disease activity, physical function, radiographic progression, and patient-reported and workability outcomes [22-27]. During long-term follow up, $\mathrm{ADA}_{\text {ORIG }}$ demonstrated sustained effectiveness and a stable safety profile for up to 10 years of treatment [28]. In small uncontrolled studies in RA patients, $\mathrm{ADA}_{\text {ORIG }}$ was shown to have a positive effect on vascular endothelial dysfunction [29] and aortic stiffness [30], suggesting a protective role against vascular comorbidity.

Despite extensive research into the effects of biological therapy in RA, little is known about its possible impact on the extent of patients' PA. This observational study was undertaken to assess the level of habitual PA and its change over time in RA patients treated with $\mathrm{ADA}_{\text {ORIG }}$ in routine clinical practice in several Central and Eastern Europe countries. The relation of PA with disease activity, physical function and certain standard sociodemographic factors was also evaluated.

\section{Methods}

\section{Study design and population}

This was a 52-week multicentre, post-marketing, observational study conducted in seven countries in Central and Eastern Europe: Bosnia and Herzegovina, Estonia, Hungary, Romania, Russia, Serbia, and Ukraine. Participating sites were medical centres experienced in the biological treatment of RA. Investigators were identified for possible participation in the study if a suitable patient population was available in their catchment area and if they were able to conduct the study in accordance with applicable legal and regulatory requirements. The study was approved by the local ethics committees of participating countries according to local law and regulations.

Eligible patients were adults $>18$ years of age with RA, diagnosed according to the 1987 American College of Rheumatology (ACR) [31] or 2010 ACR/EULAR [1] classification criteria, who had been prescribed $\mathrm{ADA}_{\mathrm{ORIG}}$ within $\leq 1$ month of study enrolment independently of inclusion into the study.
Patients received $\mathrm{ADA}_{\text {ORIG }} 40 \mathrm{mg}$ subcutaneously every other week according to the local product label and prescription and/or reimbursement guidelines and were followed for up to 12 months.

Evaluations were conducted at enrolment (baseline, Visit 0), and then at 3 months (Visit 1), 6 months (Visit 2), 9 months (Visit 3), and 12 months (Visit 4), although the actual number and timing of follow-up visits were at the investigator's discretion.

Informed consent was obtained from all study participants. Investigators have obtained appropriate institutional review board approval or have followed the principles outlined in the Declaration of Helsinki for all human or animal experimental investigations.

\section{Assessments}

RA disease activity was assessed by the Disease Activity Score-28 joints (DAS28), a composite measure of tender joint count and swollen joint count across 28 joints, Erythrocyte Sedimentation Rate (ESR), and a patient's health assessment on a $0-100 \mathrm{~mm}$ visual analogue scale $[32,33]$.

Physical function was measured by the Health Assessment Questionnaire Disability Index (HAQ-DI) [34].

Physical activity was assessed using the Short QUestionnaire to ASsess Health-enhancing (SQUASH) physical activity, a validated patient-reported questionnaire designed to measure levels of habitual PA [35]. Participants were asked to recall (for an average week in recent months) the number of days spent per week engaged in the activities, the average time (hours and minutes) spent per day on the activities, and the intensity at which they did the activity (low, moderate, high) in four domains: commuting activities (SQUASH-A), leisure time and sport activities (SQUASH-B), household activities (SQUASH-C), and activities at work or school (SQUASH-D). Based on patients' self-reported effort, activities were given an intensity score (ranging from 1 to 9) and activity scores were calculated by multiplying the total minutes of activity by the intensity score, with higher scores indicating a greater level of health-enhancing PA. The reliability and validity of SQUASH to assess daily PA have previously been demonstrated in patients with Ankylosing Spondylitis (AS) [36] and osteoarthritis [37].The instrument is available in several languages. 


\section{Statistical methods}

Analyses were performed in the population of subjects who received $\geq 1$ dose of $\mathrm{ADA}_{\text {ORIC }}$ (hereafter referred to as the study population). Descriptive statistics were used: mean \pm Standard Deviation (SD) for continuous parameters and absolute (n) and relative (\%) frequency distributions for qualitative data.

For assessments over time, analyses by visit (Visits 1-4) were performed (observed case), complemented by a Last Observation Carried Forward (LOCF) analysis based on patients with a baseline value and $\geq 1$ follow-up assessment.

The association between SQUASH scores and DAS28 $8_{\text {ESR }}$ scores, HAQ-DI scores, and sociodemographic factors was evaluated using multivariable regression analyses and correlation coefficients, supported by subgroup analyses. Pearson correlation coefficients were calculated for SQUASH scores and DAS28 $8_{\mathrm{ESR}}$ or HAQDI scores at baseline (V0), at V4-LOCF and for changes between V0 and V4-LOCF. Sociodemographic factors considered in the analysis were: age $\geq 18$ years as a continuous variable; binary variables of gender (male or female), education (less than high school, high school, more than high school), and occupation (manual or non-manual).

All statistical analyses were conducted using SAS ${ }^{\oplus}$ version 9.2 (SAS Institute, Cary, NC, USA).

\section{Results}

Patient disposition and baseline characteristics

A total of 462 patients were enrolled in the study: Bosnia and Herzegovina ( $\mathrm{n}=15 ; 3.2 \%)$; Estonia $(\mathrm{n}=11 ; 2.4 \%)$; Hungary $(\mathrm{n}=221 ; 47.8 \%)$; Romania ( $\mathrm{n}=120 ; 26.0 \%)$; Russia ( $\mathrm{n}=90 ; 19.5 \%)$; Serbia $(n=4 ; 0.9 \%)$; and Ukraine $(n=1 ; 0.2 \%)$. Two patients were excluded from analyses because no treatment with $\mathrm{ADA}_{\text {ORIG }}$ within the study was documented.

Approximately three-quarters of patients completed 12 months of $\mathrm{ADA}_{\mathrm{ORIG}}$ treatment; retention rates per study visit were $97.6 \%$ at 3 months $(\mathrm{n}=449), 88.7 \%$ at 6 months $(\mathrm{n}=408), 80.9 \%$ at 9 months $(\mathrm{n}=372)$, and $73.5 \%$ at 12 months $(\mathrm{n}=338)$. Main reasons for treatment discontinuation were investigator decision $(n=69 ; 15.0 \%)$, patient request $(\mathrm{n}=19 ; 4.1 \%)$, or adverse event $(\mathrm{n}=19 ; 4.1 \%)$.

The mean duration of $\mathrm{ADA}_{\text {ORIG }}$ treatment in the clinical practice

study population was $319 \pm 94.1$ days. Baseline demographic and clinical characteristics of the study population $(n=460)$ are summarized in Table 1 . Mean age $( \pm S D)$ was $53.9 \pm 12.1$ years, and $82.2 \%$ of patients were women. Patients were mainly employed $(38.7 \%)$ or regularly retired $(32.6 \%)$, although a relevant proportion was permanently work disabled (22.0\%). About one-quarter of the study population $(26.7 \%)$ had current comorbid cardiovascular disease. Patients had established disease (mean duration since diagnosis of $7.8 \pm 7.5$ years) and, at baseline, had highly active disease (DAS28 $8_{F S R} 6.1 \pm 1.1$ ) and moderate to severe functional impairment (HAQ-DI $1.6 \pm 0.6)$. Most patients ( $\mathrm{n}=431$; 93.7\%) were receiving other RA-specific medications at the time of inclusion, most commonly methotrexate ( $n=296 ; 64.3 \%)$.

\section{Clinical outcomes of disease activity and physical function}

The mean DAS28 $8_{\text {ESR }}$ score for disease activity decreased from $6.1 \pm 1.1$ at baseline to $2.9 \pm 1.0$ at final visit, representing an absolute change of -3.2 points. Improvement in disease activity was most pronounced within the first 3 months of $\mathrm{ADA}_{\text {ORIG }}$ treatment, followed by more gradual but continued improvement to final visit (Figure 1). Similarly, the mean physical function HAQDI score decreased from $1.6 \pm 0.6$ at baseline to $0.7 \pm 0.5$ at final visit, representing an absolute change of H́0.9 points. Improvement was most pronounced in the first 3 months of $\mathrm{ADA}_{\text {ORIG }}$ treatment, followed by more gradual but continued improvement to final visit (Figure 2).

At final visit, $37.4 \%$ of evaluable patients $(n=334)$ were in clinical remission $\left(\mathrm{DAS} 28_{\mathrm{ESR}}<2.6\right)$ and $66.2 \%$ had low disease activity (DAS2 $\left.8_{\text {ESR }}<3.2\right)$.

\section{Physical activity}

The SQUASH total score increased from baseline to final visit, showing improvement in overall habitual PA during $\mathrm{ADA}_{\mathrm{ORIG}}$ treatment. Improvements were seen in all SQUASH domains except for commuting activity (Figure 3 ).

At baseline, the highest SQUASH score was in the subdomain of leisure time and sport activities (1927.8 \pm 2673.1$)$, followed by household activities $(1792.5 \pm 1704.7)$. Scores for work and school activities and especially commuting activities were lower at $676.9 \pm 1459.5$ and $340.2 \pm$ 977.2, respectively.

The mean SQUASH total score increased from $4747.8 \pm 4203.5$ at baseline to $6063.4 \pm 4913.6$ 
Table 1. Baseline demographic and clinical characteristics of the study population ( $n=460)$.

\begin{tabular}{|l|l|}
\hline Characteristic & Value \\
\hline Age, years & $53.9 \pm 12.1$ \\
\hline Female gender & $378(82.2)$ \\
\hline RA disease duration (since diagnosis), years & $7.8 \pm 7.5$ \\
\hline Rheumatoid factor positive & $354(81.0)$ \\
\hline Anti-cyclic citrullinated peptide antibody positive & $288(70.2)$ \\
\hline Employment status & \\
\hline Employed and working outside home & $163(35.4)$ \\
\hline Employed and working at home & $15(3.3)$ \\
\hline Unemployed but seeking work & $23(5.0)$ \\
\hline Permanently work disabled & $101(22.0)$ \\
\hline Regularly retired & $150(32.6)$ \\
\hline Attending school or university & $8(1.7)$ \\
\hline Current RA-specific treatment & $431(93.7)$ \\
\hline Methotrexate & $296(64.3)$ \\
\hline Methylprednisolone & $115(25.0)$ \\
\hline Leflunomide & $110(23.9)$ \\
\hline Sulfasalazine & $88(19.1)$ \\
\hline Current comorbidity (incidence $>10 \%)$ & \\
Cardiovascular disease & $115(26.7)$ \\
\hline Other musculoskeletal disease & $52(12.8)$ \\
\hline Endocrine/metabolic disease & $45(10.8)$ \\
\hline DAS28 & $6.1 \pm 1.1$ \\
\hline HAQ-DI & $1.6 \pm 0.6$ \\
\hline SQUASH total & $4747.8 \pm 4203.5$ \\
\hline SQUASH-A, commuting activities & $340.2 \pm 977.2$ \\
\hline SQUASH-B, leisure time activities & $1927.8 \pm 2673.1$ \\
\hline SQUASH-C, household activities & $1792.5 \pm 1704.7$ \\
\hline SQUASH-D, activities at work and school & $676.9 \pm 1459.5$ \\
\hline Values are expressed as mean \pm SD or as number (per cent). & \\
\hline DAS28, Disease Activity Score-28 joints; HAQ-DI, Health Assessment Questionnaire \\
\hline Disability Index; RA, rheumatoid arthritis; SD, standard deviation; SQUASH, Short Questionnaire to Assess \\
Health-enhancing physical activity. & \\
\hline & \\
\hline
\end{tabular}

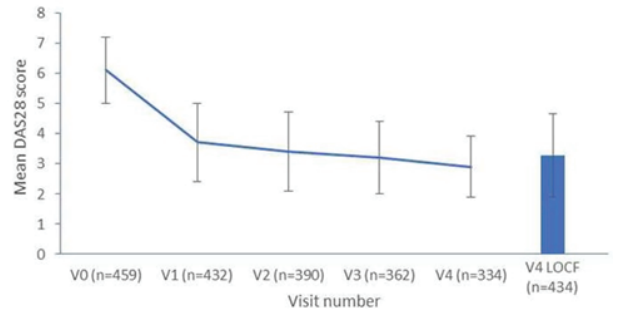

Figure 1. Change in disease activity (mean DAS28 score) during 12 months' treatment with $\mathrm{ADA}_{\mathrm{ORI}}{ }^{\circ}$ $A D A_{\text {ORIG }}$ : originator adalimumab; DAS28: Disease Activity Score-28 joints; V0: Visit 0, baseline; V1: Visit 1, 3 months; V2: Visit 2, 6 months; V3: Visit 3, 9 months; V4: Visit 4, 12 months (observed case); V4 LOCF: Visit 4, last observation carried forward.

at final visit, representing a $28 \%$ improvement in mean overall habitual PA. The overall increase in the SQUASH total score was mainly due to a $42 \%$ increase in the leisure time and sport activities subscore (V4 score of $2728.3 \pm 3401.9$ ). The subscore for work and school activities increased by $35 \%$ to $916.8 \pm 1911.0$, and the subscore for household activities increased by $14 \%$ to 2037.7 \pm 1815.2 . A small $4 \%$ decrease to $327.0 \pm 721.3$ was observed in the commuting activities sub-

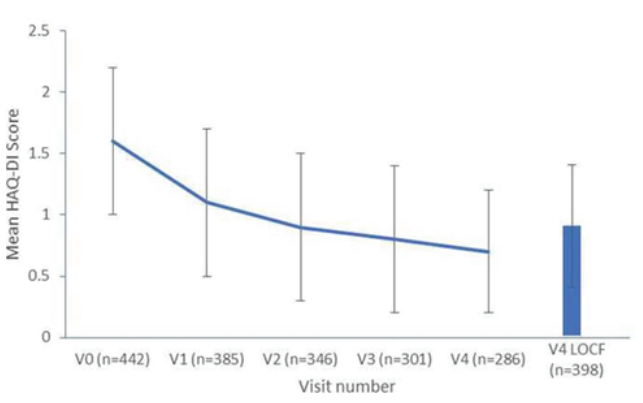

Figure 2. Change in physical function (mean HAQDI score) during 12 months' treatment with $\mathrm{ADA}_{\mathrm{ORIG}}$. $A D A_{O B I G}$ : originator adalimumab; HAQ-DI: Health Assessment Questionnaire Disability Index; V0: Visit 0, baseline; V1: Visit 1, 3 months; V2: Visit 2, 6 months; V3: Visit 3, 9 months; V4: Visit 4, 12 months (observed case); V4 LOCF: Visit 4, last observation carried forward.

score, which was the lowest score at baseline and throughout the study (Figure 3).

Relation of physical activity with disease activity and physical function

Weak negative correlations were observed between the SQUASH total score/subscores and $\mathrm{DAS}_{28_{\mathrm{ESR}}}$ scores at baseline and final visit (V4 


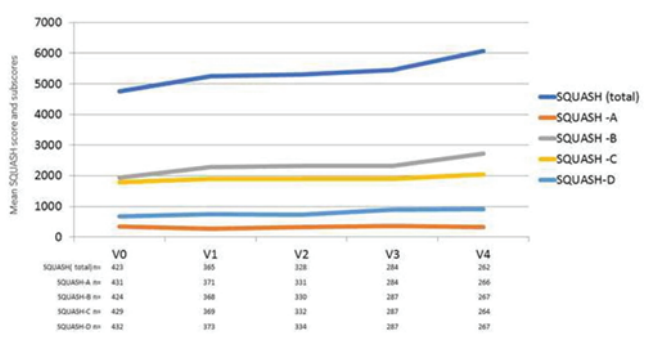

Figure 3. Change in physical activity (mean SQUASH score and subscores) during 12 months' treatment with $\mathrm{ADA}_{\mathrm{ORIG}^{\circ}} \mathrm{ADA}_{\mathrm{ORIG}}$ : originator adalimumab; SQUASH: Short QUestionnaire to ASsess Healthenhancing physical activity; SQUASH-A: commuting activities; SQUASH-B: leisure time and sport activities; SQUASH-C: household activities; SQUASH-D: activities at work and school. V0: Visit 0, baseline; V1: Visit 1, 3 months; V2: Visit 2, 6 months; V3: Visit 3, 9 months; V4: Visit 4, 12 months (observed case).

LOCF), indicating a general trend of enhanced PA with lower disease activity. Although statistical significance was reached in some cases, correlation coefficients $(\mathrm{r})$ were all below 0.3 , indicating a small effect size.

Similarly, weak negative correlations $(r=-0.1$ to -0.3) were observed between the SQUASH total score/subscores and HAQ-DI scores at baseline and final visit (V4 LOCF), suggesting a trend towards enhanced PA with improved physical function. The strongest correlation was between the SQUASH total score and HAQ-DI score at baseline $(-0.30 ; \mathrm{p}<0.0001)$.

Among sociodemographic factors only age showed a significant negative effect on the final SQUASH total score with an effect estimate of $-46.2(\mathrm{p}<0.05)$, indicating that each 1 -year increase in age above 18 years reduced the SQUASH total score by an average of 46.2 points. No substantial association with SQUASH scores was observed for gender, education or occupation.

\section{Discussion}

This prospective, multicountry, multicentre, observational study demonstrated a substantial increase in daily PA level in RA patients treated with $\mathrm{ADA}_{\text {ORIG }}$ for up to 1 year, in conjunction with improvements in standard measures of disease activity and physical function. Among evaluated sociodemographic factors (age, gender, education, occupation), only advancing age was found to have a significant negative association with the level of habitual PA at study end.

There is solid evidence for the benefits of PA in RA patients in terms of improved disease- specific and non-specific outcomes; PA may also help alleviate increased cardiovascular risk in RA [38]. However, patients with RA are generally less active than healthy controls [4-6], not only due to fear of pain, disease exacerbation and joint damage by exercising, but also because of lack of awareness and education [39]. Given the evidence for the effectiveness, feasibility and safety of regular PA, it is now formally promoted by EULAR as an integral part of standard care throughout the course of inflammatory arthritis and osteoarthritis [18].

Effective treatment to reduce disease activity and improve functional capacity might imbue RA patients with the confidence to undertake lifestyle changes, including regular PA. However, data regarding the level of PA in patients with RA are largely lacking, and even more so are data regarding possible improvement under various treatments. This study was undertaken to evaluate changes in the level of daily habitual PA in $\mathrm{RA}$ patients during treatment with $\mathrm{ADA}_{\mathrm{ORIG}}$ in clinical practice.

The self-reported SQUASH questionnaire was selected as the instrument to measure PA in our study because it is relatively simple to perform and evaluates PA in all the crucial domains (recreation and sport, household, work or school, commuting) [40]. SQUASH has been shown to have acceptable construct validity and moderate to high test-retest reliability in healthy populations $[35,41]$. It has also been tested in patients after hip arthroplasty and in patients with AS, where it was found to have a superior re-test reliability to another similar self-report questionnaire $[36,42]$. By taking into account the frequency, duration and intensity of engaging in each activity, the subscores calculated for each SQUASH domain can be compared and provide an idea of the extent of PA performed in each activity type.

At baseline, the greatest contributor to patients' overall habitual PA was time involved in leisure time and sport activities (SQUASH-B subscore; $41 \%$ ), followed closely by household activities (SQUASH-C; 38\%). The contribution to overall PA by work and school activities (SQUASH-D; $14 \%$ ) and especially by commuting activities (SQUASH-A; 7\%) was considerably lower. The mean SQUASH total score increased by $28 \%$ at study end, which corresponded with 12 months' follow-up of $\mathrm{ADA}_{\mathrm{ORIG}}$ therapy. The highest relative and absolute increase was observed in the leisure time and sport activities subscore which increased by $42 \%$ or 800 points, respectively. 
The work and school activities subscore increased by $35 \%$, but originally was much lower and the absolute increase was 240 points. The subscore for household activities increased by 14\% (245 points), while that for commuting activities, which was the lowest throughout the study, decreased by $4 \%$ (13 points) at final visit compared to baseline.

We were unable to identify any comparable data in RA patients and the minimal or clinically important difference (or similar threshold) has not yet been determined for the SQUASH total score/subscores. In a validation study involving 63 patients with AS, the mean SQUASH total score in the patient group was higher than the final score in our RA population $(7267 \pm 3453$ vs $6063 \pm 4914)$ [36]. However, among the 33 AS patients who participated in a reliability substudy, SQUASH total scores were lower for both the initial test $(5769 \pm 3360)$ and re-test $(5600 \pm$ 7540) than the final total score in our RA population [36]. Patients included in this cross-sectional study had low disease activity, minimal functional impairment, and 70\% were receiving TNF inhibitors. Based on this information in AS patients, an indirect comparison suggests that the increase in the SQUASH total score we observed in our RA patients was substantial. Interestingly, in both patient groups, the majority of PA occurred as leisure time and sports, followed by work and school activities in AS patients and by household activities in our RA group, thus reflecting the expected gender and age distribution in these respective diseases.

Clinically important improvements in disease activity and physical function were achieved with $\mathrm{ADA}_{\mathrm{ORIG}}$ therapy in our study. At the final visit, almost $40 \%$ of patients were in DAS28 clinical remission and two-thirds had low disease activity, while the mean HAQ-DI score for physical function was close to normal. However, apart from the correlation between the HAQ-DI score and SQUASH total score at baseline $(-0.30$; $\mathrm{p}<0.0001)$, no other sizeable correlations could be observed between PA and disease activity or physical function scores. This differs from the previously mentioned cross-sectional study in AS, where the SQUASH total score correlated with disease activity, physical function, spinal mobility and quality of life [36]. The absence of stronger associations in our study might be explained in part by the observational nature of study, the relatively long follow-up and missing data at final visit.
Nevertheless, all correlation coefficients in our study were in the expected direction (negative), indicating that PA generally increased with lower disease activity and improved physical function.

PA is a complex, multifactorial and multidimensional exposure variable, which complicates population-based measurement [43]. We considered possible associations between PA and the sociodemographic factors of age, gender, education and occupation. Of these, only age was found to have a significant negative relation to total PA level at study end such that, with each 1-year increase in age, the final SQUASH total score was reduced by 46 points. Van der Goes and colleagues investigated factors hindering PA levels in patients with long-standing (median 15 years) RA [44]. In a multivariate model, a higher DAS28 score, higher level of structural damage, higher age, and higher body mass index negatively influenced PA levels as measured by SQUASH, partially corresponding with our results.

One might speculate that improvements in PA may be enhanced if supported by an educational program. It is worth noting that, at the time of inclusion, more than half the study population were reported as permanently work disabled or retired, and these patients were not excluded from the analyses. The demographics of the study population likely also explains the low commuting scores, although this type of activity may be influenced by several other factors less modifiable by treatment.

The study was limited by its observational design which can introduce selection bias and provides a lower evidence level than RCTs. Moreover, the self-assessment nature of the SQUASH questionnaire is a potential source of memory and ascertainment bias. Conversely, observational studies in clinical practice provide valuable insights into the real-world disease burden, disease management and behaviour of patients with RA. As most patients were receiving medications for $\mathrm{RA}$ in addition to $\mathrm{ADA}_{\mathrm{ORIG}}$, only limited conclusions can be drawn about its effectiveness.

In conclusion, our study showed that $\mathrm{ADA}_{\mathrm{ORIG}}$ treatment in RA patients in routine clinical practice in Central and Eastern Europe led to improvements in PA, along with improvements in disease activity and physical function. Correlation was observed only between the PA total score and physical function score at baseline. Increasing age was found to be negatively associated with the overall level of PA. 
The study is registered at ClinicalTrials.gov (ClinicalTrials.gov identifier: NCT01756235).

\section{Funding}

The design, study conduct, and financial support for the study were provided by AbbVie. AbbVie participated in the interpretation of data, and in the drafting, review, and approval of the manuscript.

Medical writing support was provided by Kerry Dechant of Content Ed Net, with funding from AbbVie.

\section{Acknowledgements}

The authors wish to thank the patients, investigators, and other study staff for their contributions to the study. The authors would also like to Kerry Dechant (Content Ed Net) for her support in manuscript development.

\section{Disclosures}

Dr. Karateev has received speaker and consulting fees from AbbVie, Biocad, Bristol-Myers Squibb, Johnson \& Johnson, Novartis, Pfizer, R-Pharm, and Roche.

Dr. Rojkovich has received speaker fees and consulting fees from AbbVie, Pfizer, and Roche and has received support for clinical trials and scientific projects from $\mathrm{Ab}$ bVie, Amgen, Novartis, Sanofi, and UCB.

Dr. Codreanu has received support for clinical trials and scientific projects from AbbVie, Amgen, BMS, Egis, MSD, Pfizer, Roche, Sanofi, and UCB and has received speaker and consulting fees from AbbVie, Amgen, Angelini, AstraZeneca, BMS, Egis, MSD, Novartis, Pfizer, Richter, Roche, Sanofi, Servier, Teva, UCB, and Zentiva.

Drs. Hojnik and Nagy are employees of AbbVie, and may own AbbVie stock.

\section{Author contributions}

DK provided data collection, critical revision and final approval of the manuscript.

CC provided data collection, critical revision and final approval of the manuscript.

BR provided data collection, critical revision and final approval of the manuscript.

ON provided conception and design, analysis and interpretation of data, drafting, critical revision and final approval of the manuscript.

$\mathrm{MH}$ provided conception and design, analysis and interpretation of data, drafting, critical revision and final approval of the manuscript.

\section{References}

1. Aletaha D, Neogi T, Silman AJ et al. 2010 rheumatoid arthritis classification criteria: an American College of Rheumatology/European League Against Rheumatism collaborative initiative. Arthritis. Rheum. 62, 2569-2581 (2010).

2. Uhlig $\mathrm{T}$, Loge $\mathrm{JH}$, Kristiansen IS et al. Quantification of reduced health-related quality of life in patients with rheumatoid arthritis compared to the general population. $J$. Rheumatol. 34, 1241-1247 (2007).
3. Hansen SM, Hetland ML, Pedersen J et al. Effect of rheumatoid arthritis on long term sickness absence in 1994-2011: a Danish cohort study. J. Rheumatol. 43, 707-715 (2016).

4. Sokka T, Häkkinen A, Kautiainen $\mathrm{H}$ et al. Physical inactivity in patients with rheumatoid arthritis: data from twenty-one countries in a cross-sectional, international study. Arthritis. Rheum. 59, 42-50 (2008).

5. Munsterman T, Takken T, Wittink H. Are persons with rheumatoid arthritis deconditioned? A review of physical activity and aerobic capacity. BMC. Musculoskelet. Disord. 13, 202 (2012).

6. Hernández-Hernández V, Ferraz-Amaro I, DíazGonzález F. Influence of disease activity on the physical activity of rheumatoid arthritis patients. Rheumatology. (Oxford).53, 722-731 (2014).

7. Matcham F, Scott IC, Rayner L et al. The impact of rheumatoid arthritis on quality- of-life assessed using the SF-36: a systematic review and metaanalysis. Semin. Arthritis. Rheum. 44, 123-130 (2014).

8. Aviña-Zubieta JA, Choi HK, Sadatsafavi M et al. Risk of cardiovascular mortality in patients with rheumatoid arthritis: A meta-analysis of observational studies. Arthritis. Rheu. 59, 1690 1697 (2008).

9. Ku IA, Imboden JB, Hsue PY et al. Rheumatoid arthritis: model of systemic inflammation driving atherosclerosis. Circ. J. 73, 977-985 (2009).

10. Zegkos T, Kitas G, Dimitroulas T. Cardiovascular risk in rheumatoid arthritis: assessment, management and next steps. Ther. $A d v$. Musculoskelet. Dis. 8, 86-101 (2016).

11. Hurkmans E, van der Giesen FJ, Vliet Vlieland TP et al. Dynamic exercise programs (aerobic capacity and/or muscle strength training) in patients with rheumatoid arthritis. Cochrane. Database. Syst. Rev. 4, CD006853 (2009).

12. Cooney JK, Law RJ, Matschke V et al. Benefits of exercise in rheumatoid arthritis. J. Aging. Res. 681-640 (2011).

13. Abell JE, Hootman JM, Zack MM et al. Physical activity and health related quality of life among people with arthritis. J. Epidemiol. Community. Health. 59, 380-385 (2005).

14. Knittle KP, De Gucht V, Hurkmans EJ et al. Effect of self-efficacy and physical activity goal achievement on arthritis pain and quality of life in patients with rheumatoid arthritis. Arthritis. Care. Res. (Hoboken). 63, 1613-1619 (2011).

15. Stavropoulos-Kalinoglou A, Metsios GS, Veldhuijzen van Zanten JJ et al. Individualised aerobic and resistance exercise training improves cardiorespiratory fitness and reduces 
cardiovascular risk in patients with rheumatoid arthritis. Ann. Rheum. Dis. 72, 1819-1825 (2013).

16. Metsios GS, Stavropoulos-Kalinoglou A, Veldhuijzen van Zanten JJ et al. Individualised exercise improves endothelial function in patients with rheumatoid arthritis. Ann. Rheum. Dis. 73, 748-751 (2014)

17. Agca R, Heslinga SC, Rollefstad S et al. EULAR recommendations for cardiovascular disease risk management in patients with rheumatoid arthritis and other forms of inflammatory joint disorders: 2015/2016 update. Ann. Rheum. Dis. 76, 17-28 (2017).

18. Rausch Osthoff AK, Niedermann K, Braun J et al. 2018 EULAR recommendations for physical activity in people with inflammatory arthritis and osteoarthritis. Ann. Rheum. Dis. 77, 1251-1260 (2018).

19. Callhoff J, Weiß A, Zink A et al. Impact of biologic therapy on functional status in patients with rheumatoid arthritis-a meta-analysis. Rheumatology (Oxford). 52, 2127-2135 (2013).

20. Avci AB, Feist E, Burmester GR. Biologicals in rheumatoid arthritis: current and future. $R M D$. Open. 1,e000127 (2015).

21. Smolen JS, Landewe R, Bijlsma J et al. EULAR recommendations for the management of rheumatoid arthritis with synthetic and biological disease-modifying antirheumatic drugs: 2016 update. Ann. Rheum. Dis. 76(6), 960-977 (2017).

22. Weinblatt ME, Keystone EC, Furst DE et al. Adalimumab, a fully human anti-tumor necrosis factor alpha monoclonal antibody, for the treatment of rheumatoid arthritis in patients taking concomitant methotrexate: the ARMADA trial. Arthritis. Rheum. 48(3), 855 (2003).

23. Keystone EC, Kavanaugh AF, Sharp JT et al. Radiographic, clinical, and functional outcomes of treatment with adalimumab (a human antitumor necrosis factor monoclonal antibody) in patients with active rheumatoid arthritis receiving concomitant methotrexate therapy: a randomized, placebo-controlled, 52-week trial. Arthritis. Rheum. 50, 1400-1411 (2004).

24. van de Putte LB, Atkins C, Malaise M et al. Efficacy and safety of adalimumab as monotherapy in patients with rheumatoid arthritis for whom previous disease modifying antirheumatic drug treatment has failed. Ann. Rheum. Dis. 63, 508-516 (2004).

25. Weinblatt ME, Keystone EC, Furst DE et al. Long term efficacy and safety of adalimumab plus methotrexate in patients with rheumatoid arthritis: ARMADA 4 year extended study. Ann.
Rheum. Dis. 65, 753-759 (2006).

26. Breedveld FC, Weisman MH, Kavanaugh AF et al. The PREMIER study: A multicenter, randomized, double-blind clinical trial of combination therapy with adalimumab plus methotrexate versus methotrexate alone or adalimumab alone in patients with early, aggressive rheumatoid arthritis who had not had previous methotrexate treatment. Arthritis. Rheum. 54, 26-37 (2006).

27. Strand V, Rentz AM, Cifaldi MA et al. Healthrelated quality of life outcomes of adalimumab for patients with early rheumatoid arthritis: results from a randomized multicenter study. $J$. Rheumatol. 39, 63-72 (2012).

28. Keystone EC, Breedveld FC, van der Heijde D et al. Longterm effect of delaying combination therapy with tumor necrosis factor inhibitor in patients with aggressive early rheumatoid arthritis: 10 -year efficacy and safety of adalimumab from the randomized controlled PREMIER trial with open-label extension. J. Rheumatol. 41, 5-14 (2014).

29. Kerekes G, Soltész P, Szucs G et al. Effects of adalimumab treatment on vascular disease associated with early rheumatoid arthritis. Isr. Med. Assoc. J. 13, 147-152 (2011).

30. Vassilopoulos D, Gravos A, Vlachopoulos C et al. Adalimumab decreases aortic stiffness independently of its effect in disease activity in patients with rheumatoid arthritis. Clin. Rheumatol. 34, 359-364 (2015).

31. Arnett FC, Edworthy SM, Bloch DA et al. The American Rheumatism Association 1987 revised criteria for the classification of rheumatoid arthritis. Arthritis. Rheum. 31, 315-324 (1988).

32. van der Heijde DM, van 't Hof M, van Riel PL et al. Development of a disease activity score based on judgment in clinical practice by rheumatologists. J. Rheumatol. 20, 579-581 (1993).

33. Prevoo ML, Van 'T Hof MA, Kuper HH et al. Modified disease activity scores that include twenty-eight-joint counts: development and validation in a prospective longitudinal study of patients with rheumatoid arthritis. Arthritis. Rheum. 38, 44-48 (1995).

34. Wolfe F, Kleinheksel SM, Cathey MA et al. The clinical value of the stanford health assessment questionnaire functional disability index in patients with rheumatoid arthritis. J. Rheumatol, 15, 1480-8 (1988).

35. Wendel-Vos GC, Schuit AJ, Saris WH et al. Reproducibility and relative validity of the short questionnaire to assess health-enhancing physical activity. J. Clin. Epidemiol. 56, 1163-1169 
(2003)

36. Arends S, Hofman M, Kamsma YP et al. Daily physical activity in ankylosing spondylitis: validity and reliability of the IPAQ and SQUASH and the relation with clinical assessments. Arthritis. Res. Ther. 15, R99 (2013).

37. Makabe S, Makimoto K, Kikkawa $\mathrm{T}$ et al. Reliability and validity of the Japanese version of the short questionnaire to assess health-enhancing physical activity (SQUASH) scale in older adults. J. Phys. Ther. Sci. 27, 517-522 (2015).

38. Sveaas SH, Smedslund G, Hagen KB et al. Effect of cardiorespiratory and strength exercises on disease activity in patients with inflammatory rheumatic diseases: a systematic review and meta-analysis. Br. J. Sports. Med. 51, 1065-1072 (2017).

39. Iversen MD, Scanlon L, Frits M et al. Perceptions of physical activity engagement among adults with rheumatoid arthritis and rheumatologists. Int. J. Clin. Rheumtol. 10, 67-77 (2015).
40. van Poppel MN, Chinapaw MJ, Mokkink LB et al. Physical activity questionnaires for adults: a systematic review of measurement properties. Sports. Med. 40, 565-600 (2010).

41. Craig CL, Marshall AL, Sjöström $M$ et al. International physical activity questionnaire: 12-country reliability and validity. Med Sci. Sports. Exerc. 35, 1381-1395 (2003).

42. Wagenmakers R, van den Akker-Scheek I, Groothoff JW et al. Reliability and validity of the short questionnaire to assess health-enhancing physical activity (SQUASH) in patients after total hip arthroplasty. BMC. Musculoskelet. Disord. 9, 141 (2008).

43. Lamonte MJ and Ainsworth BE. Quantifying energy expenditure and physical activity in the context of dose response. Med. Sci. Sports. Exerc. 33(6 Suppl), S370-378, discussion S419-420 (2001).

44. van der Goes MC, Hoes JN, Cramer MJ et al. Identifying factors hampering physical activity in longstanding rheumatoid arthritis: what is the role of glucocorticoid therapy? Clin. Exp. Rheumatol. 32, 155-161 (2014). 